\title{
Performance Analysis of Power Amplifier Back-off Levels in UWB Transmitters
}

\author{
Eduard Bertran, Senior Member, IEEE, Máirtín S. O’Droma, Senior Member, IEEE, \\ Pere.L. Gilabert, and Gabriel Montoro
}

\begin{abstract}
This paper focuses on the interplay between data throughput and battery duration in UWB units, both of which the most perceptible at the user' level. The potential benefits of enlarging the power amplifier back-off (linearity) are assessed for different channel conditions according to a communication system following the IEEE 802.15. $3 a$ standard (MB-OFDM proposal). New performance results are presented which highlight the kinds of channels where an increase in transmit linearity (power amplifier back-off enlargement) yields increased data throughput, and those where it does not. Results show whether designs sacrificing power efficiency have a benefit in data rate increase or it is simply a power spoil (ineffective reduction of battery's timeof-life).
\end{abstract}

Index Terms - UWB, MB-OFDM, Power amplifiers, multipath, Back-off.

\section{INTRODUCTION}

Ultra-wideband (UWB) communications are key components of the short-range indoor segment of the future generation of wireless systems, aiming at offering very high data rates at reduced costs and low power consumption. Hence, the battery's charge time-of-life is a key aspect in UWB wireless communications. The capability to operate at reduced energy consumption it is one of the main reasons, from the consumer point of view, which will make standardized UWB the foremost option for short-range (down $10 \mathrm{~m}$ ), high speed communications (up to $480 \mathrm{Mbps}$, or $1339 \mathrm{Mbps}$, depending on different industry consortiums). In typical mobile transmitters, the delivery of the necessary transmit power is the greatest source of energy consumption, making this a principal focus of battery life lengthening research. Transmitter power efficiency is usually found to be in competition with transmitter linearity. Power amplifier

\footnotetext{
1 This work was supported by the by Spanish Government (CICYT project TEC2005-07985-C03-02), and by the EU network TARGET - "Top Amplifier Research Groups in a European Team” (IST-1-507893-NOE), http:// www.target-org.net.

E. Bertran, P.L. Gilabert and G, Montoro are with the Department of Signal Theory and Communications, Technical University of Catalonia, Spain (e-mail: \{bertran, plgilabert, montoro\}@tsc.upc.edu).

M.S.O'Droma is with the Telecommunications Research Centre, Department of Electronic and Computer Engineering, University of Limerick, Ireland (e-mail: Mairtin.ODroma@ul.ie).”
}

(PA) design is the prime example of the ongoing research into the interplay of these two competing requirements. This was an important concern in the elaboration of the Wireless Personal Area Networks (WPAN) standard IEEE 802.15.3a [1], the physical layer (PHY) of which is suitable for UWB communications.

For instance, in UWB communications, one of the considerations is the use of a power management process which allows some devices, acting as piconet coordinators, to place other devices in a power-saving mode. Lengthening battery life by lowering PA power consumption is another practical approach. In IEEE 802.15.3a the PA outputs are low, typically ranging over -12 to $7 \mathrm{dBm}$. It is this low primarily to avoid interference with other existing communication systems within the 3.1 to $10.6 \mathrm{GHz}$ band (a band where, for instance, the FCC specifies output power levels less than $-41.25 \mathrm{dBm} / \mathrm{MHz}$ ). In the alternative 2.4 $\mathrm{GHz}$ ISM band (also foreseen in IEEE standards, although shared with Bluetooth and IEEE $802.11 / \mathrm{b} / \mathrm{g}$ ) the reported values of transmit UWB power are higher, of the order of 9 $\mathrm{dBm}$. Anyway, this value is sensitive to the employed CMOS technology, which for reasons of low cost is the preferred technology.

In the development of some lower power UWB transmitters, the PA maybe omitted: with suitable mixers the modulated signal may be passed directly to the antenna [2]. At higher power levels, distributed and single-transistor based structures are both considered for PA designs. In any case the transmitter chain, with or without the PA, has to be linear enough to cope with the non-constant amplitude OFDM (or CDMA) signal, satisfying required mandatory transmitter equipment rules for maximum EVM (Error Vector Magnitude) and ACPR (Adjacent Channel Power Ratio) for the various operating conditions.

With a PA present, increased linearity is achieved by appropriate biasing of the PA operating point away from the compression region, i.e. by increasing PA back-off. This leads to increased power consumption per information quantity throughput. Normally this trade-off between linearity and power efficiency has been evaluated only at the transmitter level and independently of the characteristics of the communications channel. In indoor and mobile communications, as is the case of WPAN, the effects of channel multipath and low speed Doppler need to be considered also to completely evaluate transmitter performance in the context of overall communications system 


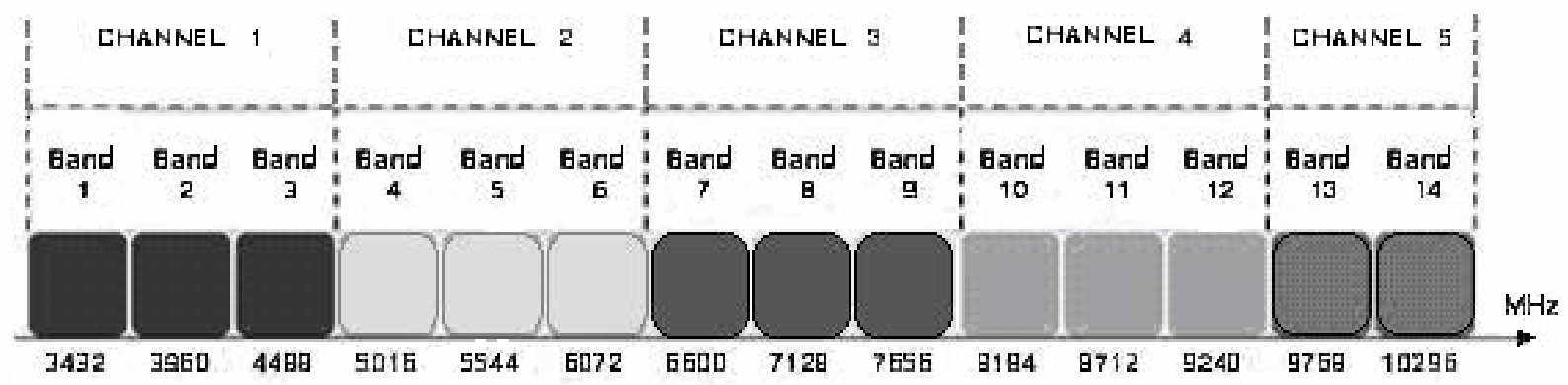

Fig. 1. UWB Spectrum (MB-OFDM).

performance $[3,4,5]$. This approach yields more accurate and reliable conclusions in regard to guidelines on the most suitable PA linearity degree. This is particularly so for those situations where multipath and/or Doppler can counter (or simply, hide) the benefits of increasing the PA linearity, with the consequent sacrifice in battery charge duration but with no gain in data throughput. In business parlance, there is a need to detect and obviate noncompetitive operating conditions for UWB transmitters.

The linearity-efficiency compromise is here assessed considering some disturbances (noise, multipath and Doppler) in the non-linear channel (i.e. including the PA nonlinearities as part of the channel). The conclusions are obtained from studies based on the IEEE 802.15.3a MultiBand Orthogonal Frequency Division Multiplexing (MBOFDM) PHY layer proposed by the MB-OFDM Alliance (also, MBOA).

\section{Phy Layer IEeE 802.15.3a}

IEEE has two main standards for WPANs: IEEE 802.15.3a and IEEE 802.15.4a. The first aims at high bitrate transmission (up to $10 \mathrm{~m}$ ), while the latter enlarges the application range at the expense of reducing the transmission speed. Since the main economic benefits of UWB radio are expected in WPANs, these standards have become the focus for the main applications for UWB technology business.

IEEE 802.15.3a task group considered basic data rates of 11, 22, 33, 44 and 55 Mbps. These were extended in later proposals: MBOA seeks $480 \mathrm{Mbps}$ (equivalent to wired USB 2.0 devices [6]) using QPSK modulations, while the DS-UWB consortium anticipates 1339 Mbps from 4-BOK modulation. These data-rates are justified by the estimated rates required for various high bandwidth WPAN applications (e.g. H.323/T.120 Video Conference and Home Theater), ranging 40 - $188 \mathrm{Mbps}$, [7].

The rest of the paper will consider only MBOA's IEEE 802.15.3a proposal, due to its mature status. Also, despite the fact that the IEEE standard may operate both in the 2.4 $\mathrm{GHz}$ ISM band and within an unlicensed spectrum from 3.168 to $10.56 \mathrm{GHz}$, we will only consider the band from $3.168 \mathrm{GHz}$ to $4.752 \mathrm{GHz}$. This band corresponds to the band-group 1 within the five UWB spectrum band-groups (encompassing a total of 14 sub-bands), Fig.1, and is split in three sub-bands of $528 \mathrm{MHz}$, [8]. It is considered as optimal for initial deployments and $1^{\text {st }}$ generation devices of the MB-OFDM solution. Up to four Simultaneously Operating Piconets, SOP, may operate in the band. According to the power spectrum mask, the interference of signals in any band into adjacent bands must be less than $20 \mathrm{dBr}$.

In MB-OFDM, bands and tones may be dynamically turned-off to satisfy different national or regional regulations. Besides, with OFDM, stopping the generation of some specific tones is possible. This can be required to protect singular frequencies, such as the specific tones in the $3 \mathrm{GHz}$ band used for radio-astronomy in Japan. OFDM is also an efficient solution against multipath: IEEE 802.15.3a has to be robust for multipath occurring with a delay spread of up to $60.6 \mathrm{~ns}$, [7].

IEEE 802.15.3a standard uses FEC coding at different coding rates (11/32,1/2,5/8 and 3/4), IFFT (128 points), different spreading rates $(4,2$ and 1$)$ and QPSK modulation (BPSK is also considered), [9] and [10].

\section{Communication ChanNel}

\section{A. Multipath}

Multipath is unavoidable in indoor communications, the intended WPAN environment. Domestic obstacles (furniture, stairs, walls,...) produce several variable-length paths for transmit waves which combine at the receiver antenna usually destructively. Depending on the presence or absence of LOS (Line of Sight) between the transmitter and receiver antennas, the main kinds of multipath follows models of Rice (LOS), Rayleigh (NLOS, fast fading), or lognormal statistics (NLOS) in case of shadow (slow) fading.

In the simulation results presented in this paper, four different usual types of channel models were used. The Power Delay Profiles (PDP) of these UWB channels are presented in [7] and [9].

\section{B. Doppler}

For a single tone the Doppler effect is similar to a frequency jitter. However, in presence of different rays (multipath), the joint Doppler effects (Doppler spread), on 
recombination at the receiver antenna, may cause serious degradations. The nominal Doppler frequency is defined by:

$$
f_{d}=v / \lambda
$$

where $f_{d}$ is the Doppler frequency, $v$ is the receiver (or transmitter) speed and $\lambda$ is the wavelength. The maximum Doppler frequency spread (spectral broadening) is given by $B_{d}=2 f_{d}$.

In OFDM modulations, as it is critical to maintain good orthogonally between sub-carriers, Doppler spread may be a significant source of impairment: even small phase deviations among the different OFDM sub-carriers may produce demodulation errors.

\section{SIMULATION RESULTS}

Using the Matlab/Simulink software, the transmitted signal is QPSK modulated, with a 5/8 rate FEC convolution code, and OFDM modulation using 122 subcarriers (128 points FFT). This scenario, compliant with the IEEE 802.15.3a standard, corresponds to the $200 \mathrm{Mbps}$ case of the MB-OFDM proposals. The receiver includes a Viterbi decoder.

The PA selected is a MMIC HMC326MS8G (Hittite) which operates at MB-OFDM first band-group (Fig. 1). Thus the amplifier is suitable for $1^{\text {st }}$ generation devices It operates in the band $3-4.5 \mathrm{GHz}$ with a gain of $21 \mathrm{~dB}$, output saturation power, Psat, of $28 \mathrm{dBm}$ and third order intercept point of $38 \mathrm{dBm}$. The static distortion curves (AM-AM, AM-PM) were analytically approximated with the Saleh model.

The Doppler spread simulation has been based on the time-efficient SoS (Sum of Sinusoids) method.

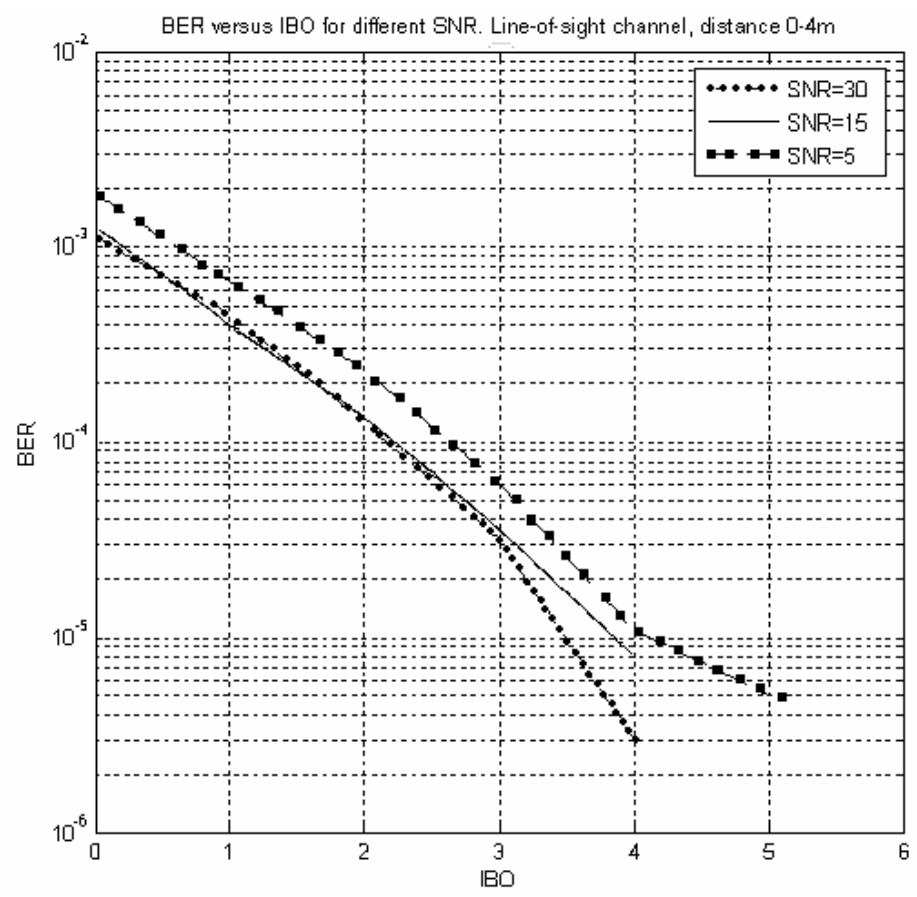

Fig. 2. BER versus IBO for different SNR. Line-of-sight (LOS) channel, distance $0-4 \mathrm{~m}$.
Fig. 2 shows the BER (Bit Error Rate) as a function of PA input back-off, IBO $\{\mathrm{IBO}=10 \log (\mathrm{Psat} / \mathrm{Pin})\}$ where Psat is saturated power) on an LOS channel with RMS delay of $5.28 \mathrm{~ns}$. Results are computed for different values of the Signal-to-Noise Ratio (SNR). Fig. 3 presents the situation for NLOS with RMS delay of 25ns.

Comparing curves in Figs. 2 and 3 to those presented in [9], it is possible to conclude that a distance decrement of $1 \mathrm{~m}$ is equivalent, in BER, to an IBO augmentation of $2 \mathrm{~dB}$. Also comparing these figures with [9], it is seen that the improvement obtained by increasing $\mathrm{E}_{\mathrm{b}} / \mathrm{N}_{0}$ by $1 \mathrm{~dB}$ is similar to increasing IBO by $2 \mathrm{~dB}$ (LOS), or $3 \mathrm{~dB}$ (NLOS). In Fig. 3 (NLOS) the BER curves become flatter at low SNR values. Hence, in noisy environments, augmentation of the IBO (and consequently, the power consumption) has a lower effect on the BER than in low noise channels. Even, at very low SNR, the BER is not modified by increasing IBO. This fact contrasts with Fig. 2 (LOS), where BER is always sensitive to IBO, with a sensitivity value practically independent of the SNR ratio.

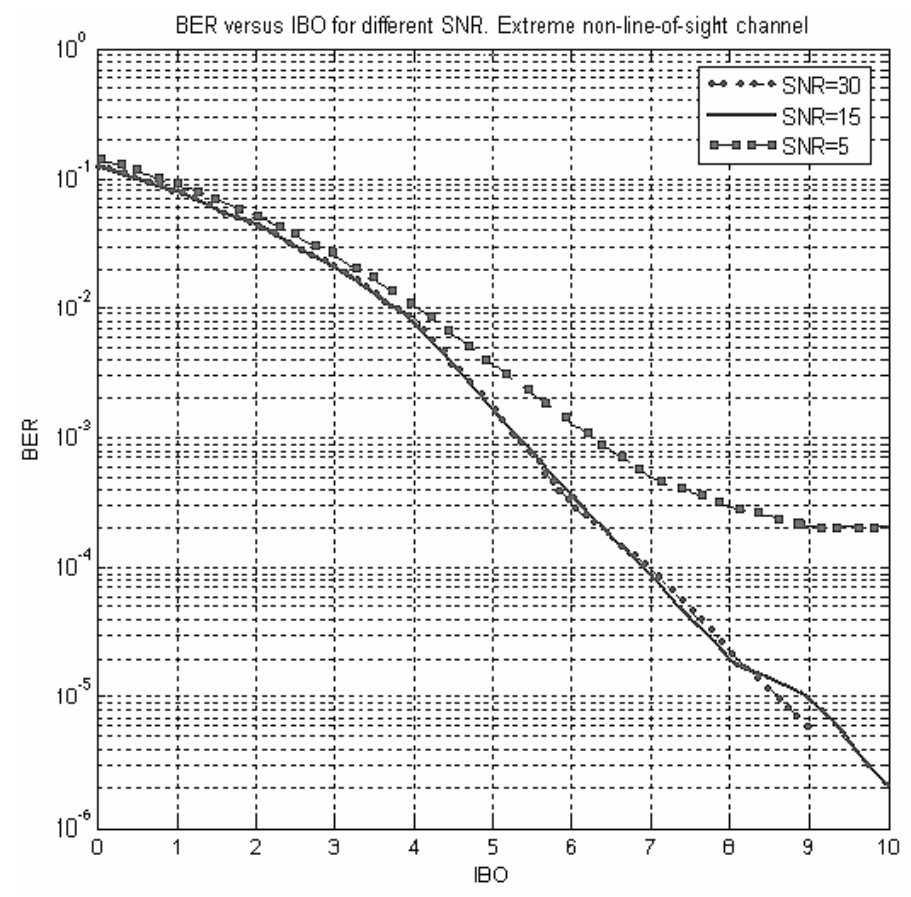

Fig. 3. BER versus IBO for different SNR. Extreme non-lineof-sight (NLOS) channel, distance $0-4 \mathrm{~m}$.

In Fig. 4 the SNR is fixed to $15 \mathrm{~dB}$, and BER is compared for different kinds of channels. It may be noticed that the BER sensitivity to IBO increments is similar for all the channels. Also, an interesting aspect is revealed in Fig. 4: in a NLOS channel, UWB devices work better at larger distances $(4-10 \mathrm{~m})$ than at short ranges $(0-4 \mathrm{~m})$. Besides, for a fixed BER of $10^{-5}$, the required IBO shows differences of up to $5 \mathrm{~dB}$ depending on the LOS or NLOS channel conditions. 


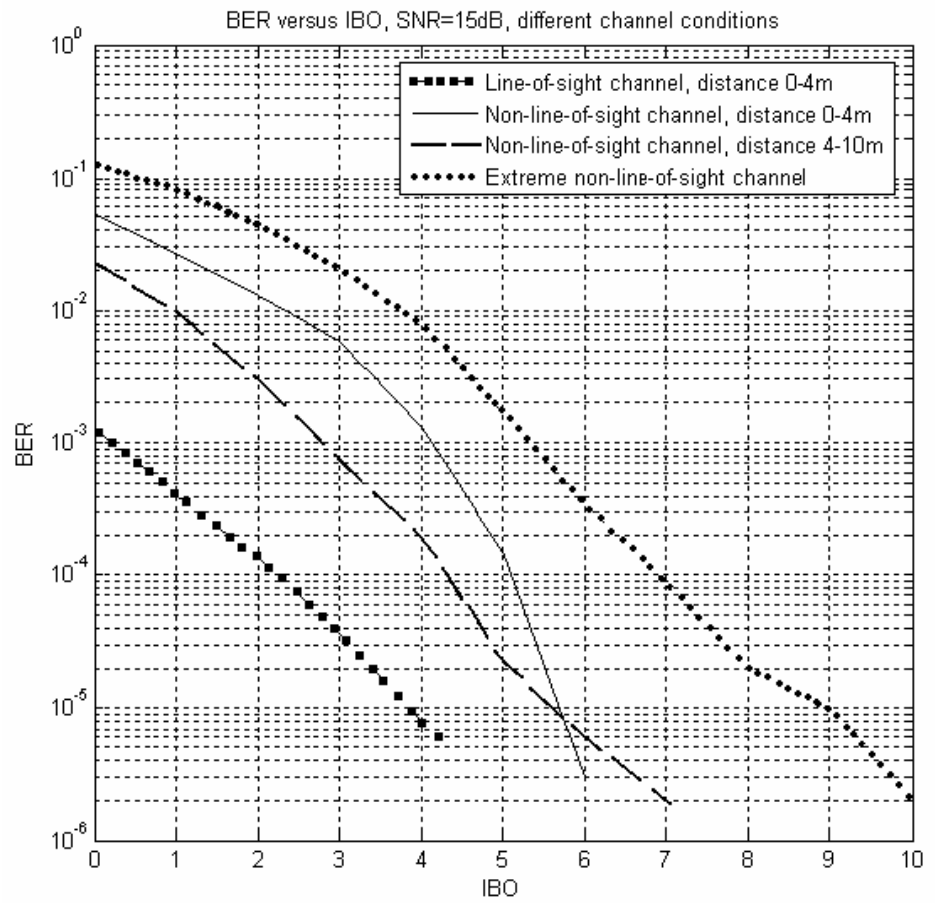

Fig. 4. BER versus IBO for different channel situations $(\mathrm{SNR}=$ 15dB).

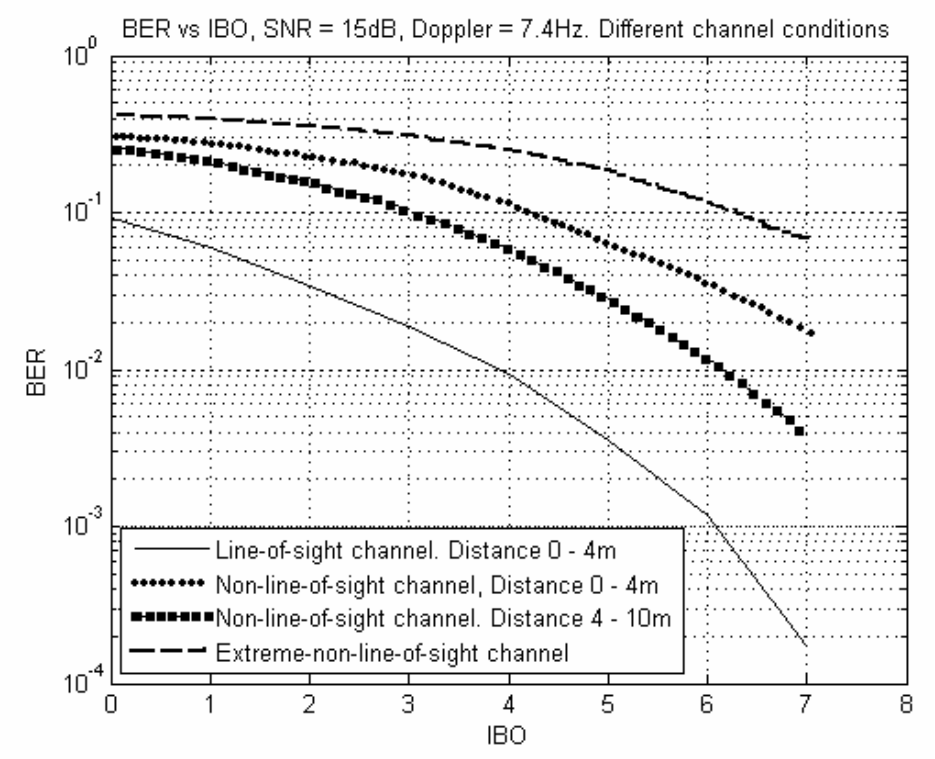

Fig. 5. BER versus IBO for different channels and low speed Doppler $(2 \mathrm{Km} / \mathrm{h}, \mathrm{SNR}=15 \mathrm{~dB})$.

Finally, Fig. 5 shows the variation of BER versus IBO with a Doppler of $7.4 \mathrm{~Hz}$. Notice, comparing Figs. 4 and 5, that Doppler causes worse BER than channel AWGN. Besides, the slope of curves in presence of Doppler (Fig. 6) is lower, indicating a minor sensitivity of BER versus IBO increments.

\section{CONCLUSION}

In MB-OFDM technology, BER is highly sensitive to nonlinearity effects, especially those caused by the PA in the transmitter. However, for the IEEE 802.15 3a MBOFDM UWB communication system, and taking the channel conditions into consideration, it is found that the main impediment to increasing data throughput is Doppler shift. This causes higher BER degradation than that due to noise or multipath. In such scenarios increasing transmit power, e.g. by PA backoff reduction, does not help. This is so even if linearization or signals clipping schemes are employed to reduce nonlinearity impairment effects. Only in LOS, low-noised, Doppler-free scenarios, such as those usually encountered in home WPAN environments, will the benefits of varying transmit power level impact on BER and/or data throughput and thus where linearization of transmitters over the full dynamic power range gains importance.

\section{ACKNOWLEDGEMENT}

The authors wish to thank Juan Murillo for his valuable work on computer simulation.

\section{REFERENCES}

[1] IEEE 802.15 WPAN High Rate Alternative PHY Task Group 3a (TG3a), http://grouper.ieee.org/groups/802/15/pub/TG3a.html.

[2] A. Cidronali, I Magrini, M Camprini, E. Bertran et al, "TX system level analysis by accurate behavioural modelling of RF building blocks," Proceedings European Microwave Week, Paris, October 2005.

[3] B.M. Donlan, D.R. McKinstry, R.M. Buehre "The UWB indoor channel: large and small scale modeling", IEEE Trans. Wireless Commun., vol 5, n 10, Oct. 2006 pp: 2863 - 2873.

[4] R.C. Qiu, "Generalized time domain multipath channel and its application in ultra-wideband (UWB) wireless optimal receiver part III: system performance analysis", IEEE Trans. Wireless Commun., vol 5, n 10, Oct. 2006 pp: 2685 - 2695.

[5] I.R. Capoglu., Li Ye, A. Swami, A. "Effect of Doppler spread in OFDM-based UWB systems", IEEE Trans. Wireless Commun., vol 4, n 5, Sept. 2005 pp: 2559 - 2567.

[6] Gary Breed, "Wireless USB Uses Ultra Wideband (UWB) for High Data Rate", High Frequency Electronics, September 2005.

[7] K. Mandke, H. Nam, L. Yerramneni, and C. Zuniga, "The evolution of UWB and IEEE 802.15.3a for very high data rate WPAN," EE 381K-11 Wireless Communications UWB Group, The University of Texas and Austin, May 62003. http://www.winlab.rutgers.edu/ diwu/Document/Reference/wpan AnalUT.pdf

[8] Wimedia Alliance, MultiBand OFDM Update and Overview", Sept 2004, http://wimedia.org/

[9] A. Batra, et. al. "TI Physical Layer Proposal for IEEE 802.15 Task Group 3a," IEEE P802.15-03/142r2. July, 14, 2003 http://focus.ti.com/lit/an/s1la139/slla139.pdf

[10] Multiband OFDM Physical Layer Specification (Rev. 1.0), January 14, 2005, WiMedia Alliance. 


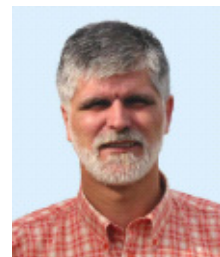

Eduard Bertran (SM'02) received the Ingeniero and Doctor Ingeniero degrees in Telecommunication Engineering, both from the Technical University of Catalonia (UPC), Barcelona, Spain, in 1979 and 1985, respectively. He became a lecturer at UPC in 1980, and joined the department of Signal Theory and Communications (TSC) in 1987, where he is currently a Full Professor. He has been the head of studies of the TSC department and Associate Dean in different telecommunications schools (curricula director \& laboratories). His research interests include communication electronics, wireless communications, control, signal processing and circuit theory, and he has collaborated with industries and other research institutions in different national and international projects and networks. He acts as Secretary of the Spanish Section of the IEEE.

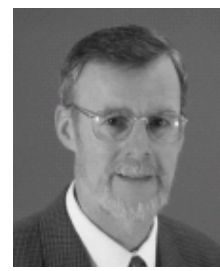

Máirtín S. O'Droma B.E., Ph.D, C.Eng., FIEE (SM) received his bachelor's and doctoral degrees from the National University of Ireland in 1973 and 1978 respectively. He is a Senior Academia and Director of the Telecommunications Research Center at the University of Limerick, Ireland. Current activities include founding partner of TARGET - Top Amplifier Research Groups in a European Team -, a European Union Network of Excellence, EU-FP6-NoE-IST507893, 2004-2008, www.targetnet.org, and heading up the RF power amplifier linearization research strand. $\mathrm{He}$ was a founding partner of ANWIRE, EUFP5-IST-38835, 2002-2004, and of two European "Cooperation in the field of Science and Technology" Research Actions (COST Actions 285 \& 290) focused on wireless communications. Previous posts held by Dr. O'Droma include lecturer in the University College Dublin and in the National University of Ireland, Galway, Director of Communications Software Ltd and ODR Patents Ltd. His research interests include: complex wireless telecommunication systems simulation and behavioral modeling, linearization \& efficiency techniques in multimode, multicarrier broadband nonlinear microwave and $\mathrm{mm}$-wave transmit power amplifiers; smart adaptive antenna arrays and MIMO channels; wireless network and protocol infrastructural innovations and new paradigms

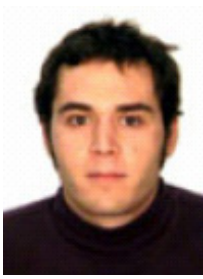

Pere Ll. Gilabert received the M. Sc. Degree in Telecommunication Engineering from the Technical University of Catalonia (UPC), Barcelona, in 2002, and did his Master thesis at the University of Rome "La Sapienza". He is currently a Ph.D. candidate and his research is focused in the study of power amplifier linearization techniques in the department of Signal Theory and Communications (TSC) at UPC. His research interests include control, communication electronics, signal processing and circuit theory. He is currently lecturing at the Escola Politcnica Superior de Castelldefels (EPSC) as Assistant Professor.

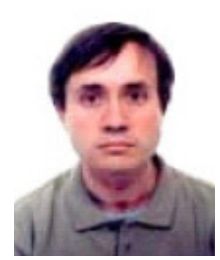

Gabriel Montoro was born in Barcelona (Spain). He received the M.S. degree in Telecommunication Engineering from the Technical University of Catalonia (UPC) in 1989 and did his final project at the area of Adaptive Control. He joined the department of Signal Theory and Communications (TSC) in 1991, where he is currently an Associate Professor. He received the Ph.D. degree of Telecommunications in 1996. His research interests include control, consumer and communications electronics, topics in which he has collaborated in both private and public funded projects. 